\title{
Heat affected zones and oxidation marks in fibre laser-oxygen cutting of mild steel
}

\author{
S.O. Al-Mashikhi ${ }^{1,3}$, J. Powell ${ }^{2}$, A. Kaplan ${ }^{2}$ and K.T.Voisey ${ }^{3}$ \\ 1. Salalah College of Technology, Engineering Department, Salalah ,Oman. \\ 2. Lulea University of Technology, Dept. of Applied Physics and Mechanical \\ Engineering, SE 97187 Lulea, Sweden.
}

3. Faculty of Engineering, University of Nottingham, Nottingham, NG7 2RD, UK.

\begin{abstract}
The effect of cutting speed and sheet thickness on surface oxidation and heat affected zones (HAZ) has been investigated for laser-oxygen cutting of mild steel sheet with a fibre laser. Optical and scanning electron micrographs were used to determine the extent of surface oxidation and HAZ from plan and cross-sectional views respectively. The HAZ is consistently wider at the bottom of the cut compared to the HAZ at the top of the cut. With increasing speed, the width of the HAZ at the top of the cut decreases whereas the HAZ width at the bottom of the cut generally increases. No simple, direct relationship between HAZ width and surface oxidation was seen. However, it is possible to state that in each case considered here, the HAZ would be completely removed if they are machined back by a depth equal to the extent of the surface oxidation.
\end{abstract}

Keywords: fibre laser; fiber laser; laser-oxygen cutting; heat affected zone; oxidation mark 


\section{Introduction}

Laser-oxygen cutting of mild steel is a highly successful industrial profiling method which has been under continuous development since its invention in 1967 [1]. In laser cutting a melt front is generated that extends through the thickness of the material. Melt is flushed from the cut front by an assist gas, thereby advancing the cut front. In laseroxygen cutting the oxygen assist gas promotes oxidation of the molten melt. The energy released by the oxidation reaction acts as an additional heat source, typically contributing approximately half of the required energy and thereby enhancing the cutting process [2, 3].

During laser cutting material near the cut edge experiences a severe thermal cycle as the laser passes by. The rapid heating and cooling can induce microstructural changes. The region referred to as the heat affected zone (HAZ) consists of material that has not melted, yet has undergone heat induced microstructural modification whilst in the solid state. Martensitic transformations, carbide dissolution [4] and grain growth [5] are features that may be observed in the HAZ. The modified microstructure of the HAZ influences its material properties and performance, including hardness and corrosion behaviour [5]. In laser cutting of steel, martensitic transformations result in the HAZ having an increased hardness. The presence of a surface layer of brittle martensitic material raises concerns about crack initiation and propagation $[6,7]$. There is therefore interest in the dimensions of the HAZ.

In laser-oxygen cutting darkening caused by the oxidation of material bordering the cut that was heated in the presence of the oxygen cutting jet is frequently observed. The 
oxidation marks correspond to the localised presence of thin oxide films on the surface of the cut material. Oxidation marks are easily visible immediately after laser cutting whereas sectioning and metallurgical sample preparation is required for examination of the HAZ.

This work examines the oxidation marks and HAZ generated by fibre laser-oxygen cutting of mild steel. The possibility of inferring the extent of the HAZ from the oxidation marks, thereby eliminating the requirement for destructive sectioning, is investigated.

\section{Experimental Method}

Laser-oxygen cutting was carried out using a YLR-2000 2kW IPG fibre laser. A $200 \mu \mathrm{m}$ diameter process fibre that produces a focussed spot size of $\sim 200 \mu \mathrm{m}$ was employed. Throughout this work the laser was focussed on the top surface of the material being cut. The laser power was fixed at $1000 \mathrm{~W}$ and a range of cutting speeds, up to $7000 \mathrm{~mm} \mathrm{~min}^{-1}$ were used. The range of possible cutting speeds is thickness dependent, hence the different thicknesses have been cut with different speeds. In each case at least 5 speeds have been used, these have speed increments of $500 \mathrm{~mm} \mathrm{~min}^{-1}$. A 2 bar oxygen jet was delivered to the cutting zone, co-axial with the laser beam.

The material cut was mild steel plate with thicknesses of 1,2,3 and $4 \mathrm{~mm}$. During cutting the plate was clamped in a fixture mounted on a CNC table which was translated under the stationary laser to produce cuts. The cutting fixture holds the plate above a cavity in order to allow free flow of the oxygen gas, as well as ensuring that molten material can be properly flushed from the cut. 
Oxidation marks (Figure 1) were measured from optical images of the as-cut plate, marks from both top and bottom surfaces were measured. Results presented are the averages of 6 individual measurements.

In order to determine HAZ width, samples were cut from the cut edge and then mounted, ground and polished to produce a cross-section of the cut edge. These samples were etched with $2 \%$ nital and then imaged using optical microscopy. The HAZ width was measured in three different locations: the top, middle and bottom regions of the cut. The top region was regarded as the top $15 \%$ of the plate thickness, similarly the middle regions was the central $15 \%$ and the bottom region the bottom $15 \%$ of the plate thickness. There is a degree of subjectivity in defining the extent of the HAZ, the dotted line in Figure 1 Upper surface of $2 \mathrm{~mm}$ mild steel cut at a speed of $4500 \mathrm{~mm} \mathrm{~min}^{-1}$. The darkening of the material adjacent to the cut edge due to oxidation is visible, the arrows indicate the start and end points of a typical oxidation mark measurement.

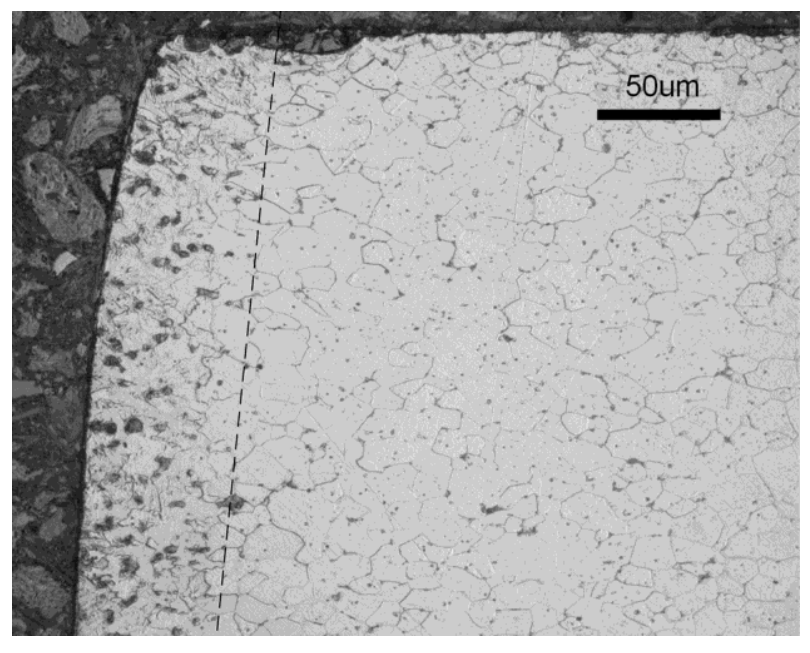

Figure 2 indicates what was regarded as the HAZ in the current work. In the HAZ widths reported, each data point is the average of 5 individual measurements. Error bars 
corresponding to the standard deviation of the measurements have been plotted, though in some cases these are hard to see due to the relatively small numerical values of the errors.

To determine if there is a simple correlation between the HAZ and oxidation mark measurements, the oxidation mark results were divided by the corresponding HAZ results: upper surface oxidation mark measurements were divided by top HAZ results, lower surface oxidation mark measurements were divided by bottom HAZ results.

Rosenthal's three dimensional solution was used to model thermal profiles and to obtain a theoretical estimate of the HAZ for comparison with the experimental results. Thermal profiles were determined at the material surface $(\mathrm{z}=0)$, in the direction extending perpendicularly from the cutting direction (y direction). A power of $1000 \mathrm{~W}$ was used. The HAZ width was determined from the Rosenthal thermal profiles. It was assumed that sufficiently fast cooling occurs so that all material with a maximum temperature between $1537^{\circ} \mathrm{C}$ and $800^{\circ} \mathrm{C}$, i.e. temperature between the melting point and an approximation of the boundary of the austenitic phase, is converted into martensite.

\section{Results}

\subsection{HAZ width.}

The figures below show the top, middle and bottom heat affected zone depths for 1, 2, 3 and $4 \mathrm{~mm}$ thick samples at a laser power of $1000 \mathrm{~W}$ over a range of speeds.

There is an overall trend for the HAZ to increase with material thickness. For each thickness cut, the top HAZ is smallest, and the bottom HAZ largest. The top HAZ gets gradually shallower as cutting speed increases. The variation of the bottom HAZ is more 
complex, it can be deep or shallow at low speeds but is then rather constant at intermediate speeds before increasing at high speeds.

\subsection{Surface oxidation marks.}

Figures $7-10$ show the oxidation marks measured on fibre laser-oxygen cut mild steel. Both upper and lower oxidation marks increase in size with material thickness. Lower oxidation marks are consistently larger than the upper oxidation marks. Increasing speed results in a general decrease in the size of the oxidation marks on the upper cut surface. The behaviour of the lower oxidation marks is more varied, showing little variation with speed for the $1 \mathrm{~mm}$ material, increasing with speed for the $2 \mathrm{~mm}$ material and decreasing with increasing speed for both the 3 and $4 \mathrm{~mm}$ material.

\subsection{Correlation of HAZ width and surface oxidation marks.}

The oxidation mark/HAZ ratios are plotted in Figures 11-14. All calculated ratios are great than 1 , indicating that the oxidation marks are consistently larger than the corresponding HAZ results. The ratios are largely unaffected by speed for the 1 and $2 \mathrm{~mm}$ thickness material, remaining approximately constant. As material thickness increases the oxidation mark/HAZ ratios tend to decrease with increased cutting speed.

\subsection{Correlation of HAZ width with Rosenthal predictions.}

The results from the Rosenthal equation for the top surface $(\mathrm{z}=0)$ of the $2 \mathrm{~mm}$ material cut at a range of speeds are shown in Figure 15. The HAZ decreases significantly as cutting speed increases. 


\section{Discussion}

The HAZ observations are consistent with previously published results [8-12], and all simply result from how heat is transferred from the flowing melt to the material surrounding the cut.

The laser cutting process generates a molten cut front through the material thickness. As the cutting progresses the cut front moves through the material and molten material leaves the cutting zone by flowing down the cut front and exiting through the bottom of the cut. The HAZ is created due to heat being conducted from the hot melt into the material surrounding the cut. If thicker materials are cut more melt has to flow out of the cut zone, increasing the amount of heat that will be conducted into the surrounding material from the melt which will increase the extent of the HAZ.

The top HAZ is the shallowest in all cases. This is due to a relatively small proportion of the total melt moving past this region of the cut front. At the bottom of the cut the solid material is heated by the passage of all the melt from the cut because all the melt must exit through the bottom of the cut zone. Hence there is more heat conducted into the material surrounding the bottom of the cut, resulting in a larger HAZ at the bottom of the cut.

As cutting speeds are increased the melt exits the cut more rapidly and hence has less time to conduct heat into the surrounding material and so the HAZ is expected to decrease, as is seen for the top HAZ, and as is seen in the Rosenthal predictions. This is not observed for the bottom HAZ because as the speed increases, the temperature of the cut front rises. The increased temperature will tend to increase the heat flow and can 
counteract the speed related decrease in HAZ, resulting in a relatively constant bottom HAZ depth over a range of speeds. At the highest speeds this balance breaks down and the bottom HAZ becomes larger. This is due to a decrease in the efficiency of melt removal at the highest speeds. This decrease in melt removal efficiency means that melt removal is incomplete and some melt is left behind at the bottom of the cut. This retained melt, or dross (Figure 16), adheres to the underside of the cut plate where it cools, passing additional heat into the solid material, resulting in a large HAZ. The Rosenthal predictions do not take such melt flow effects into account and hence, in this case, greatly overestimates the effect of cutting speed on HAZ width. The results from the Rosenthal equation show a much more significant effect of cutting speed than is seen in the experimental results. The absolute values of the modelled HAZ widths are all $150 \%$ or more greater than the experimentally measured values. This highlights the role that melt flow plays in determining the actual HAZ and shows a need to take melt flow properly into account when modelling the thermal field at the cut front. Melt flow is an additional pathway by which heat can leave the cut front, hence neglecting this will overestimate the extent of heating of the HAZ. It should also be noted that further modelling of the thermal fields must also incorporate the heat input from the oxidation reaction.

There are striking similarities between the trends in the HAZ and oxidation mark results. For both measurements the magnitude increases with material thickness, the measurements at the top or upper region of the cut are consistently greater than those from the bottom or lower region and the magnitude of the top or upper measurement decreases as cutting speed increases whereas the behaviour of the bottom or lower measurement is more complex. 
The similarities are because both oxidation marks and the HAZ are generated by heating. Hence the arguments above regarding how melt flow and heat conduction affect the HAZ can also be used to explain the corresponding trends in the oxidation mark results.

The oxidation/HAZ ratio results show that there is no simple direct correlation between the HAZ and oxidation mark results. However it is clear that the oxidation marks are consistently greater than the extent of the HAZ, with oxidation marks typically extending for twice the extent of the HAZ.

In certain laser cutting applications the HAZ may need to be removed in order to improve fatigue life. Our results show that if the cut edge is machined back to the extent of the visible oxidation mark, then the entire, interior and therefore not externally visible, HAZ will have been removed. The results presented above show that the oxidation mark exceeds the HAZ by at least $50 \%$ in each case. Hence the proposed simple and easy to implement rule of thumb for HAZ removal has an in built safety margin that guarantees complete HAZ removal.

It should be noted that our results to date only apply to mild steel and that further experiments are required on other materials and steels with higher hardenabilities before being able to generalise these conclusions.

\section{Conclusions}

There are striking similarities between the variation in oxidation marks and HAZ with cutting speed and material thickness. These have been explained in terms of heat flow and are consistent with previously reported results. 
Melt flow at the cut front has an important role in determining the extent of the HAZ.

The results show that there is no simple, direct relationship between the oxide mark width and HAZ. However, it is possible to say that, for the mild steel used in this work, all the HAZ would be removed from these samples if they were machined back by a depth equal to the oxidation mark. This suggestion would need to be confirmed over a wide range of materials, thicknesses and laser types before it could be put forward in a generalised form useful to industry.

\section{Acknowledgements}

Thanks are due to Mr Ahmed Alias who carried out some of the HAZ and oxidation zone measurements as part of an undergraduate individual research project supervised by $\mathrm{Dr}$ K.T. Voisey and S.O. Al-Mashikhi in the Department of Mechanical, Materials and Manufacturing Engineering, University of Nottingham. 


\section{References}

[1] A. B. J. Sullivan and P. T. Houldcroft, British Welding Journal 14 (1967) 443.

[2] A. Ivarson, J. Powell and C. Magnusson, Welding in the World 30 (1992) 116.

[3] A. Ivarson, J. Powell and C. Magnusson, J Laser Applications 3 (1991) 41.

[4] P. S. Sheng and V. S. Joshi, J Mat Proc Tech 55 (1995) 879.

[5] H.-H. Huang, W.-T. Tsai and J.-T. Lee, Electrochimica Acta 41 (1996) 1191.

[6] S. L. Mannan and M. Valsan, Int J of Mechanical Sciences 48 (2006) 160.

[7] S. E. Webster and P. H. Bateson, Materials Science and Technology 9 (1993) 83.

[8] M. Manohar, R. L. Bodnar, R. I. Asfahani, N. Chen and C. Huang, J Laser Applications 17 (2005) 211.

[9] W. O'Neill and J. T. Gabzdyl, Optics and Lasers in Engineering 34 (2000) 355.

[10] M. Sundar, A. K. Nath, D. K. Bandyopadhyay, S. P. Chaudhuri, P. K. Dey and D. Misra, Int J Adv Manuf Technol 40 (2009) 865.

[11] J. Powell, A. Ivarson, J. Kamalu, G. Broden and C. Magnusson, "ICALEO 92"

(Orlando, FL, 1993) p. 433.

[12] N. Rajaram, J. Sheikh-Ahmad and S. H. Cheraghi, Int J Machine Tools \& Manufacture 43 (2003) 351. 


\section{Figure captions}

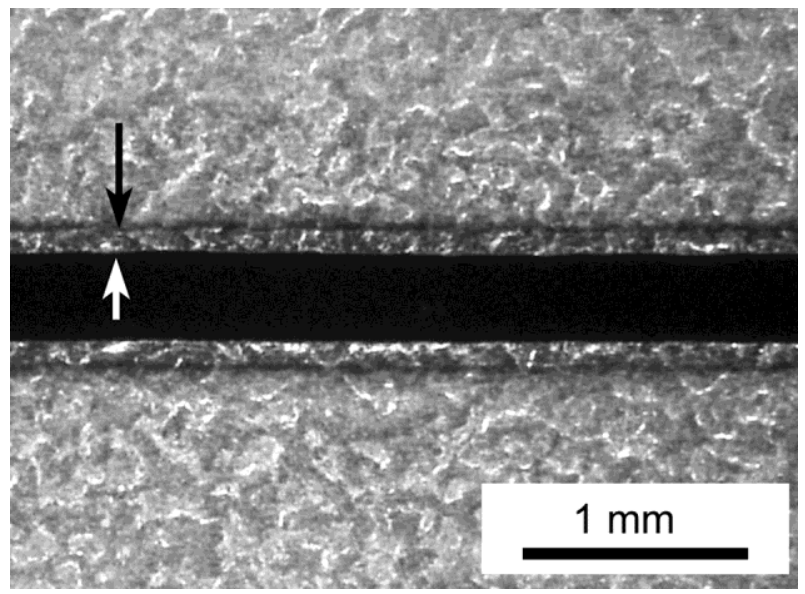

Figure 1 Upper surface of $2 \mathrm{~mm}$ mild steel cut at a speed of $4500 \mathrm{~mm} \mathrm{~min}^{-1}$. The darkening of the material adjacent to the cut edge due to oxidation is visible, the arrows indicate the start and end points of a typical oxidation mark measurement.

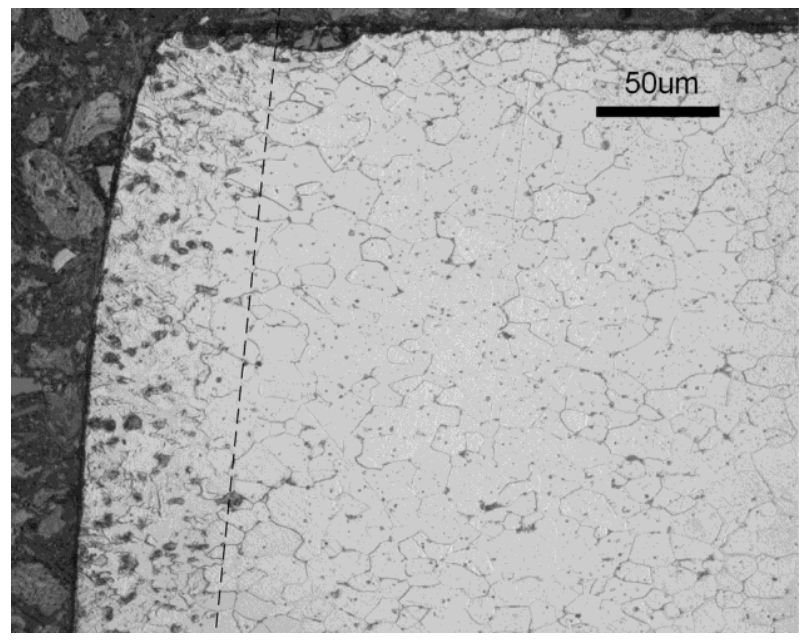

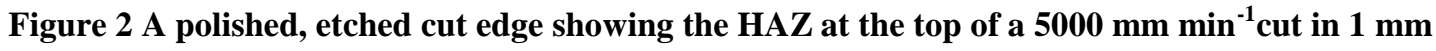
mild steel plate. The dotted line represents the boundary of the HAZ which was used to measure its depth from the cut surface. 


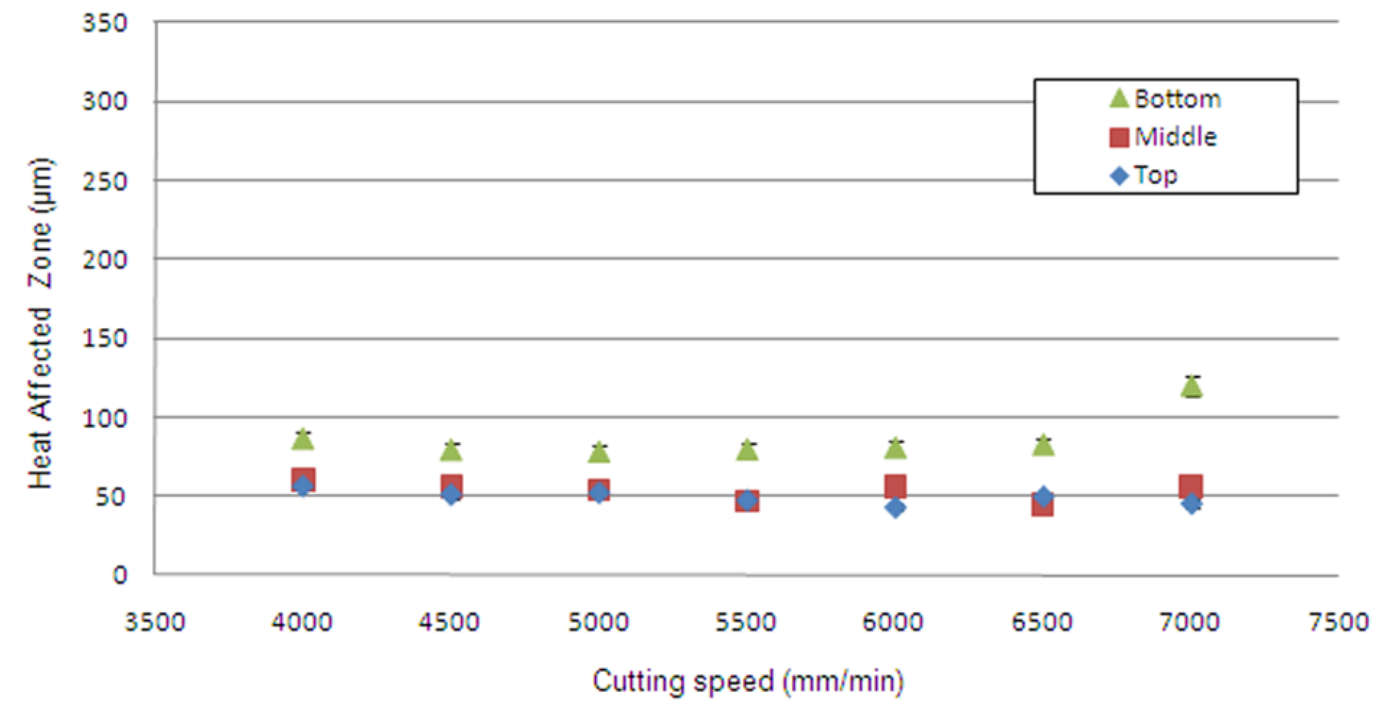

Figure 3 HAZ depth at top, middle and bottom of the cut edge for $1 \mathrm{~mm}$ thick mild steel cut at a range of speeds at a laser power of $1000 \mathrm{~W}$.

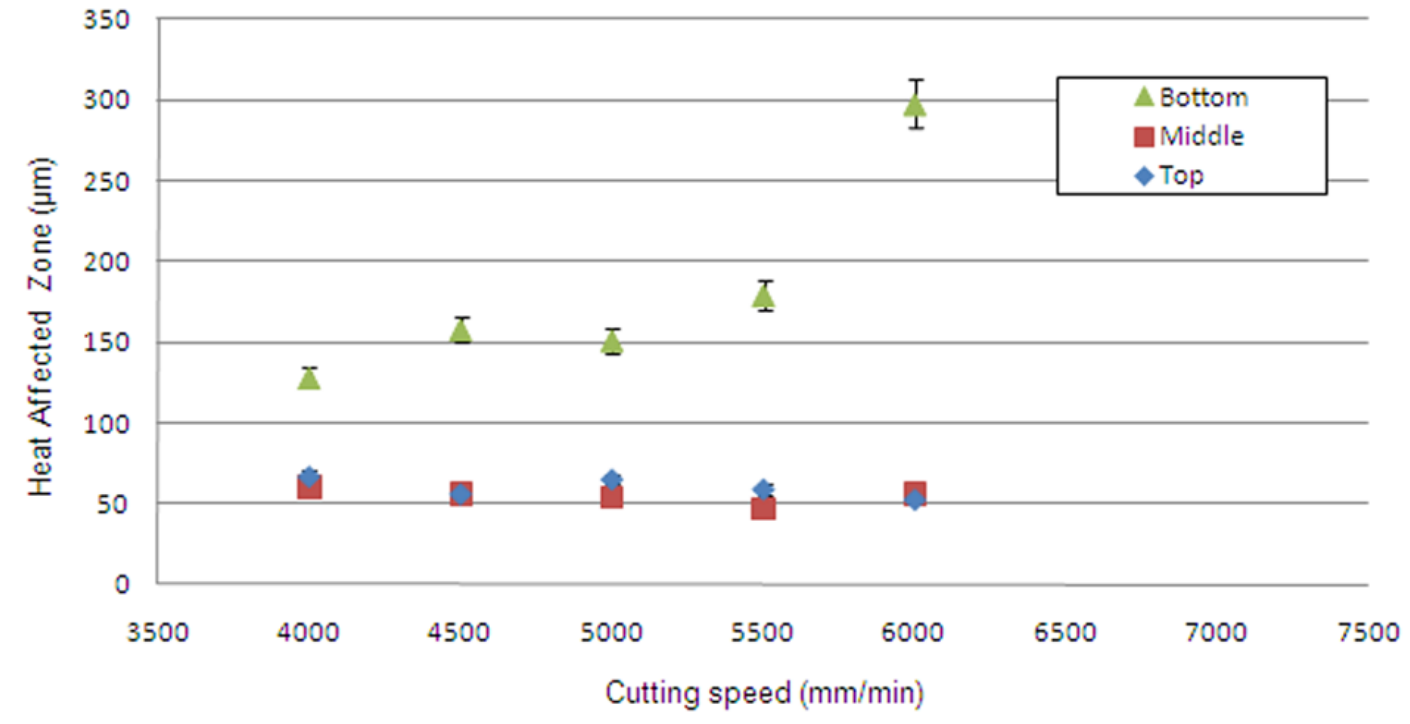

Figure $4 \mathrm{HAZ}$ depths at the top, middle and bottom of the cut edge for $2 \mathrm{~mm}$ thick mild steel cut at a range of speeds at a laser power of $1000 \mathrm{~W}$. 


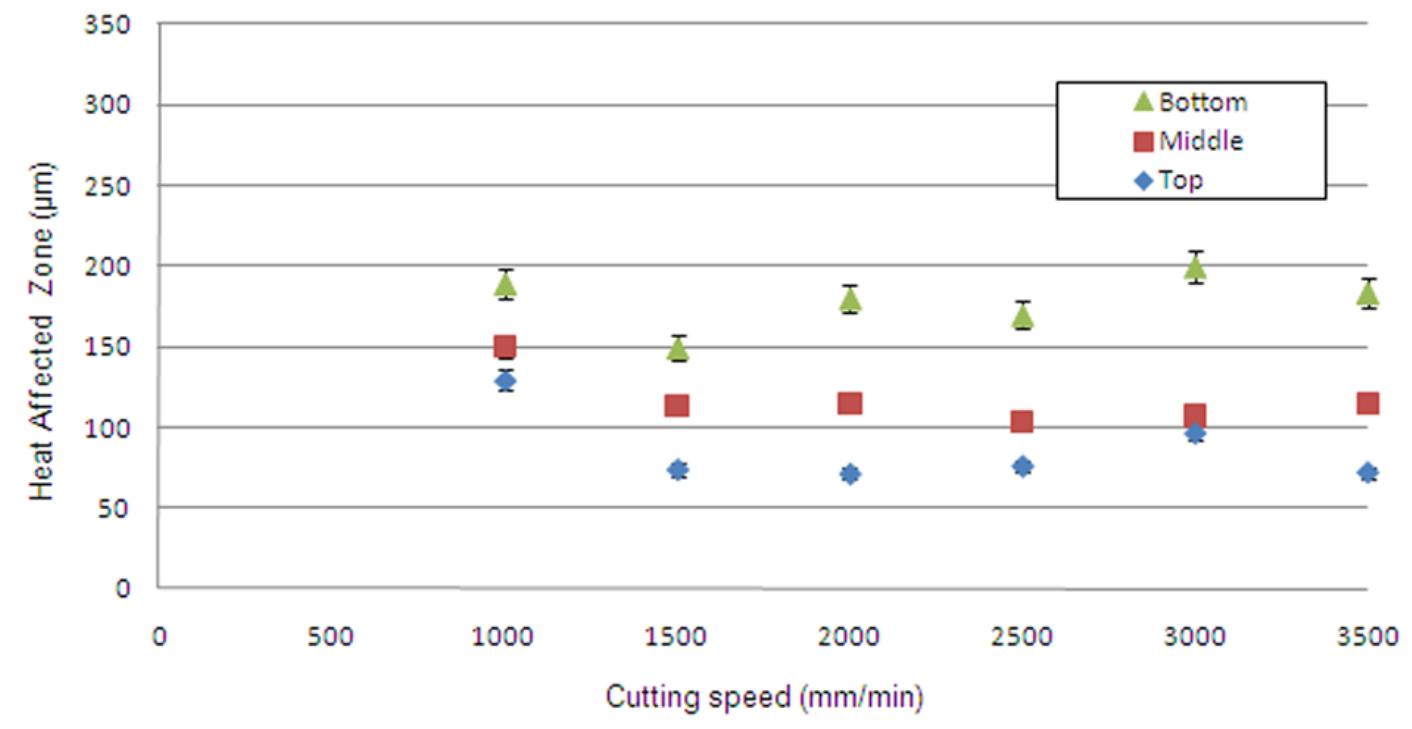

Figure 5 HAZ depths at the top, middle and bottom of the cut edge for $3 \mathrm{~mm}$ thick mild steel cut at a range of speeds at a laser power of $1000 \mathrm{~W}$.

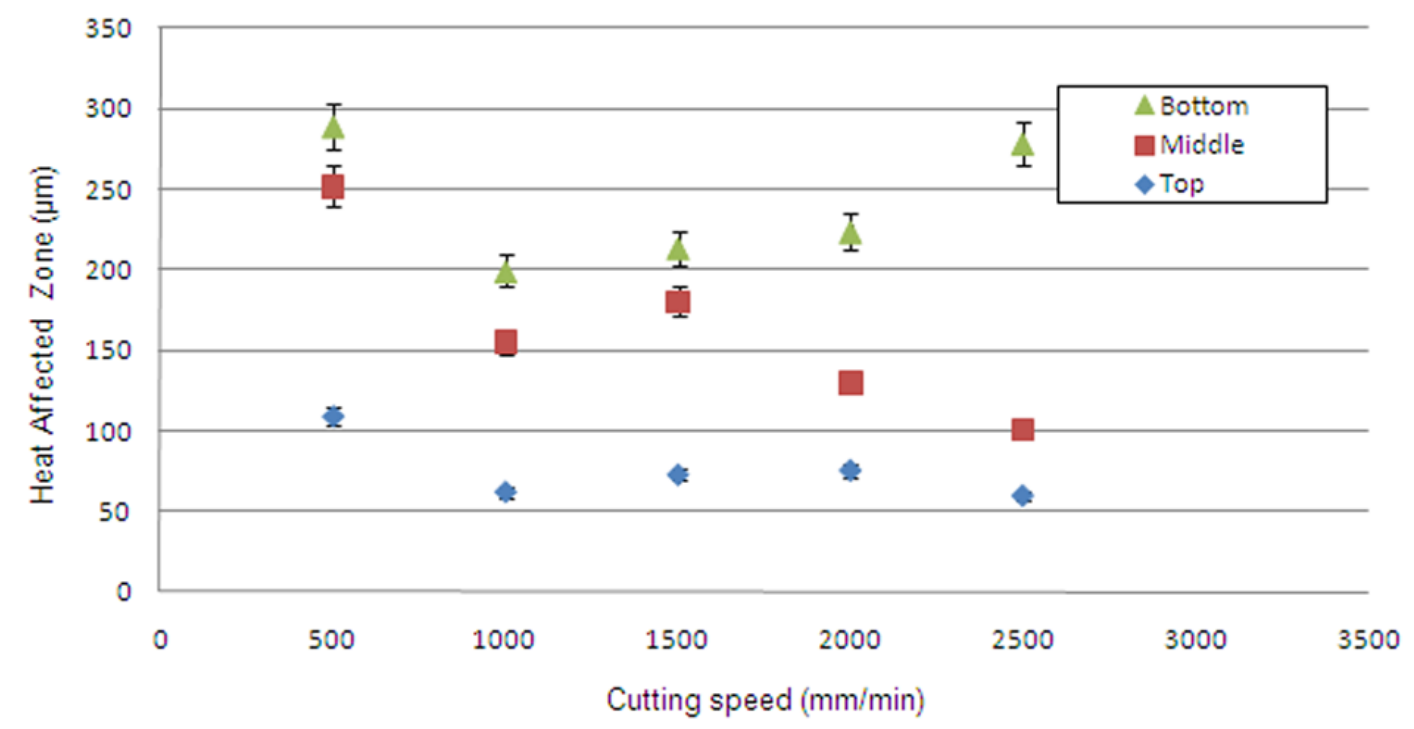

Figure $6 \mathrm{HAZ}$ depths at the top, middle and bottom of the cut edge for $4 \mathrm{~mm}$ thick mild steel cut at a range of speeds at a laser power of $1000 \mathrm{~W}$. 


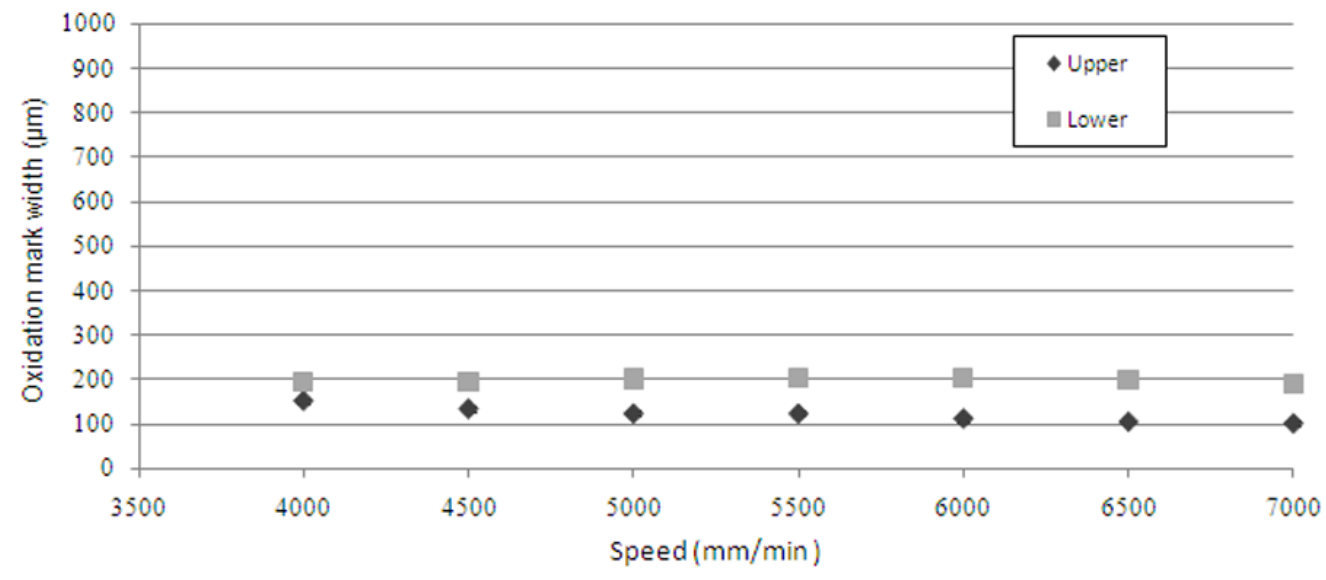

Figure 7 The widths of the upper and lower surface oxidation marks as a function of cutting speed for the $1 \mathrm{~mm}$ thick material.

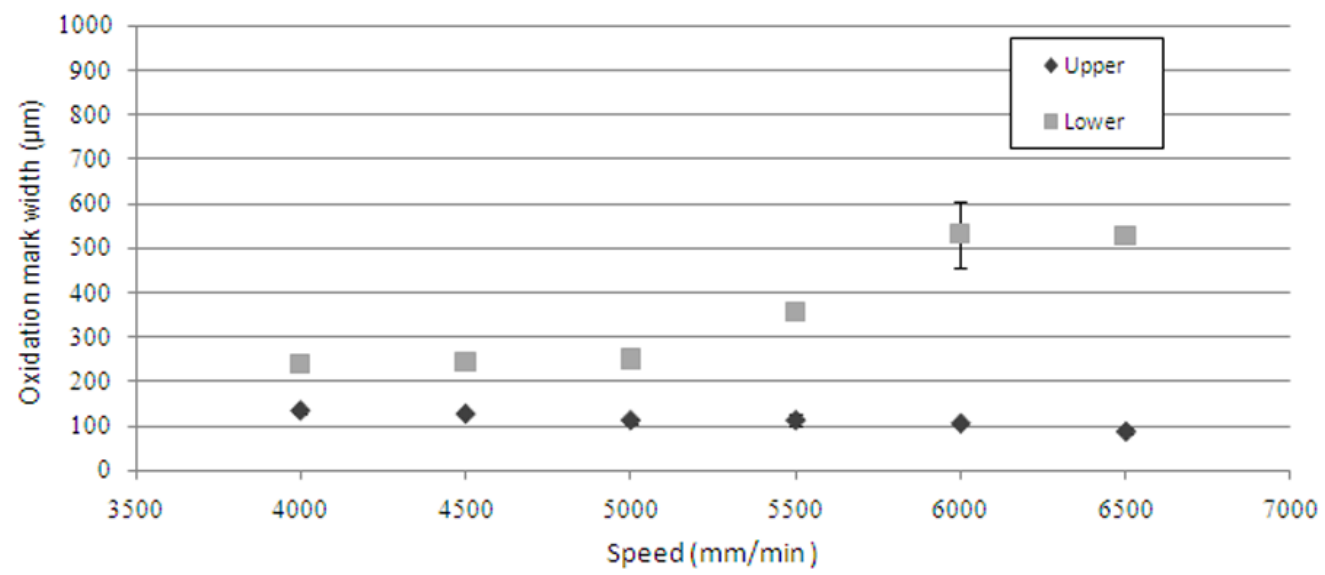

Figure 8 The widths of the upper and lower surface oxidation marks as a function of cutting speed for the $2 \mathrm{~mm}$ thick material. 


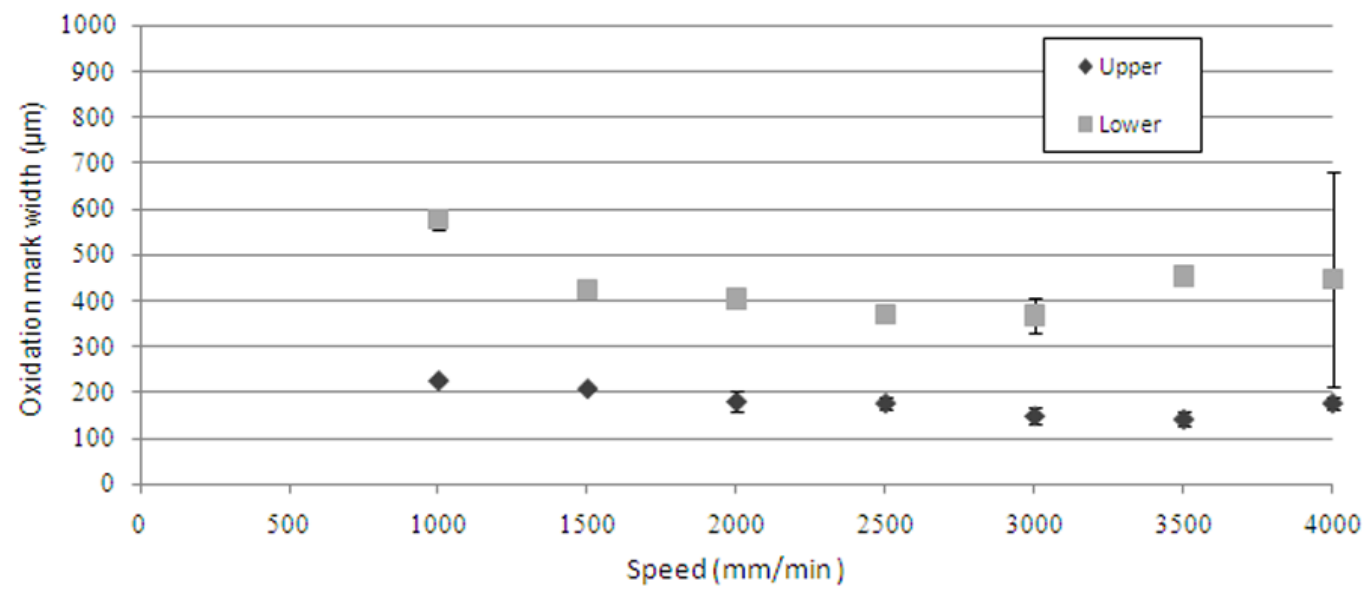

Figure 9 The widths of the upper and lower surface oxidation marks as a function of cutting speed for the $3 \mathrm{~mm}$ thick material.

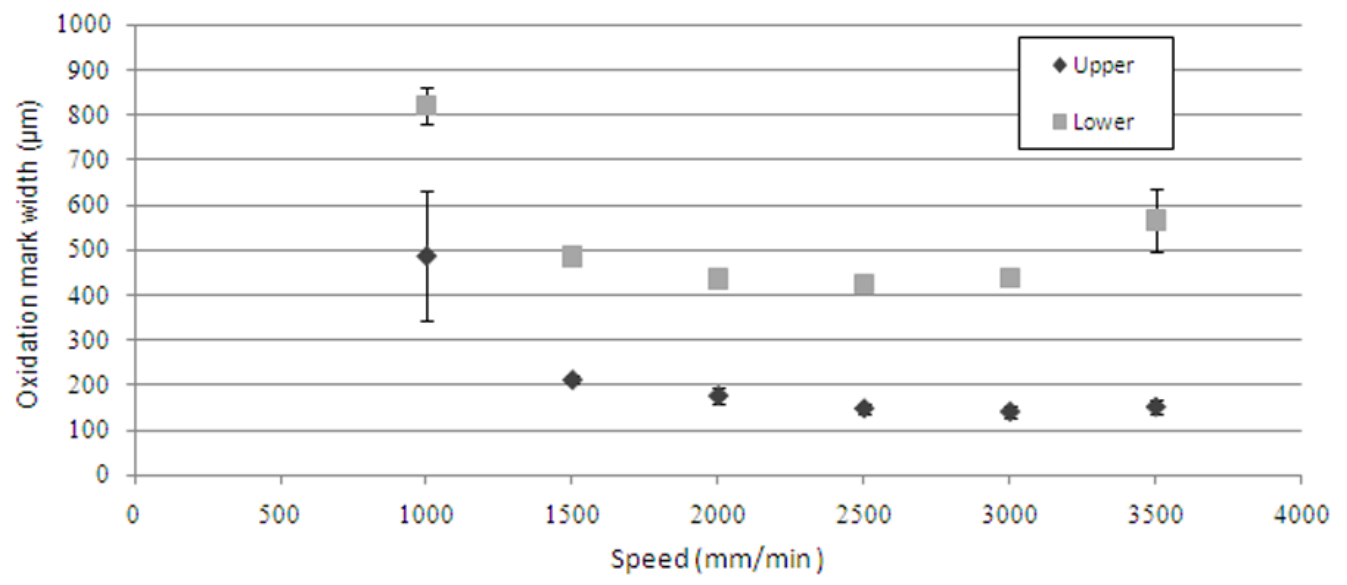

Figure 10 The widths of the upper and lower surface oxidation marks as a function of cutting speed for the $4 \mathrm{~mm}$ thick material. 


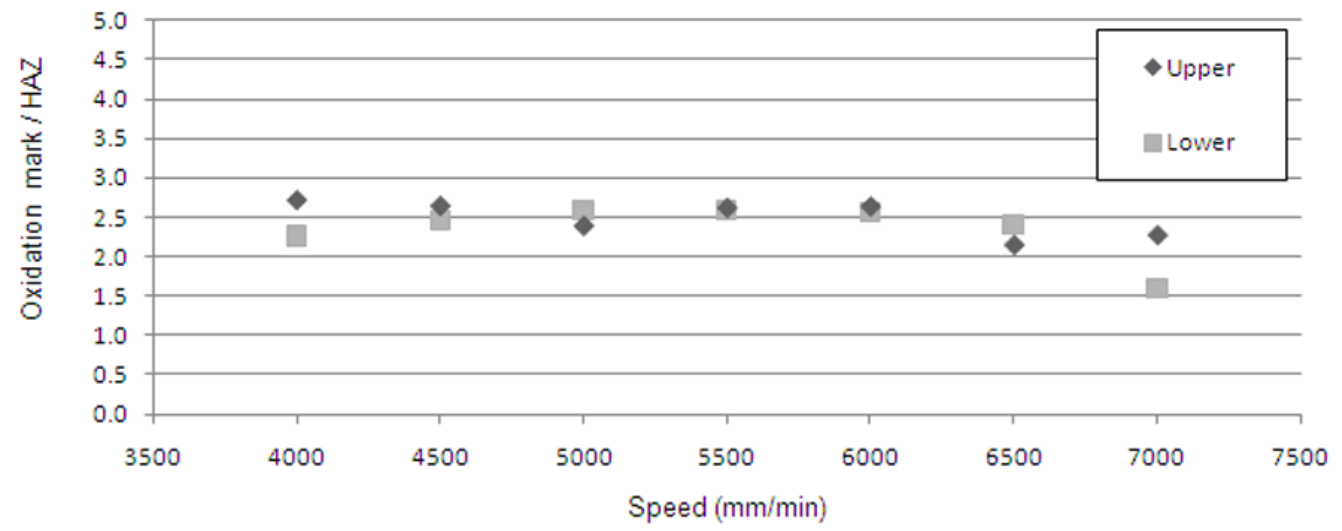

Figure 11 The ratio of the oxidation mark to the HAZ width for $1 \mathrm{~mm}$.

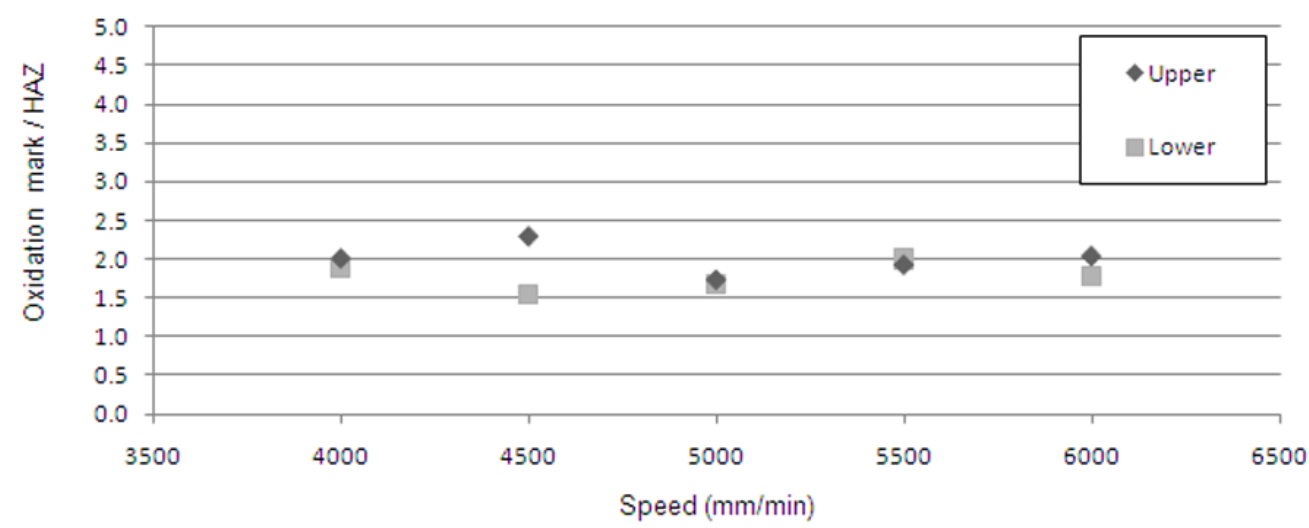

Figure 12 The ratio of the oxidation mark to the HAZ width for $2 \mathrm{~mm}$ mild steel.

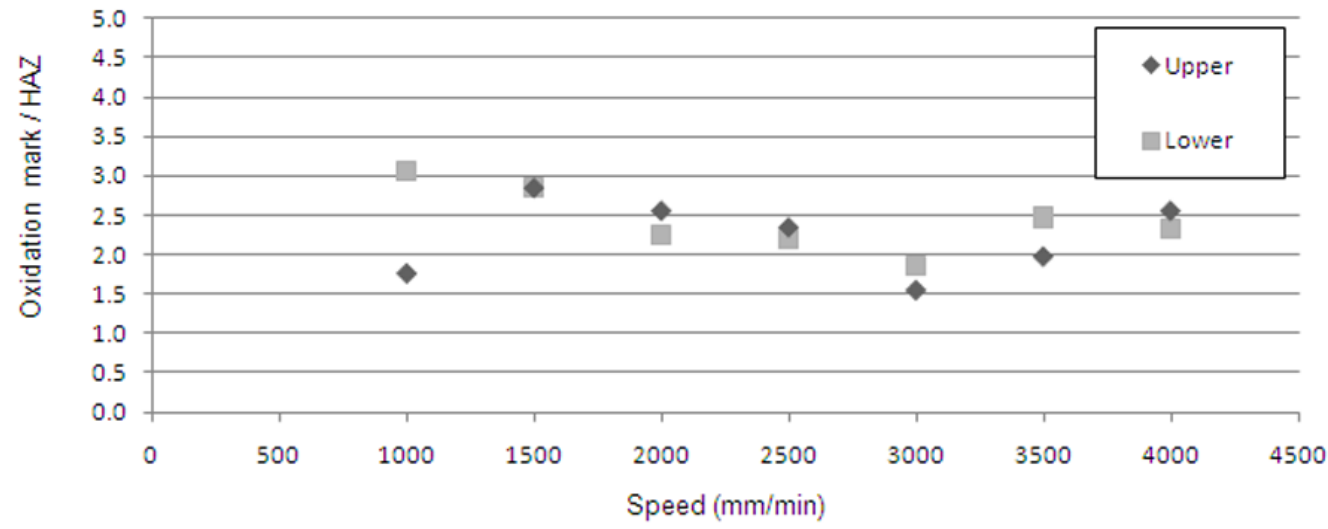

Figure 13 The ratio of the oxidation mark to the $\mathrm{HAZ}$ width for $3.0 \mathrm{~mm}$ mild steel. 


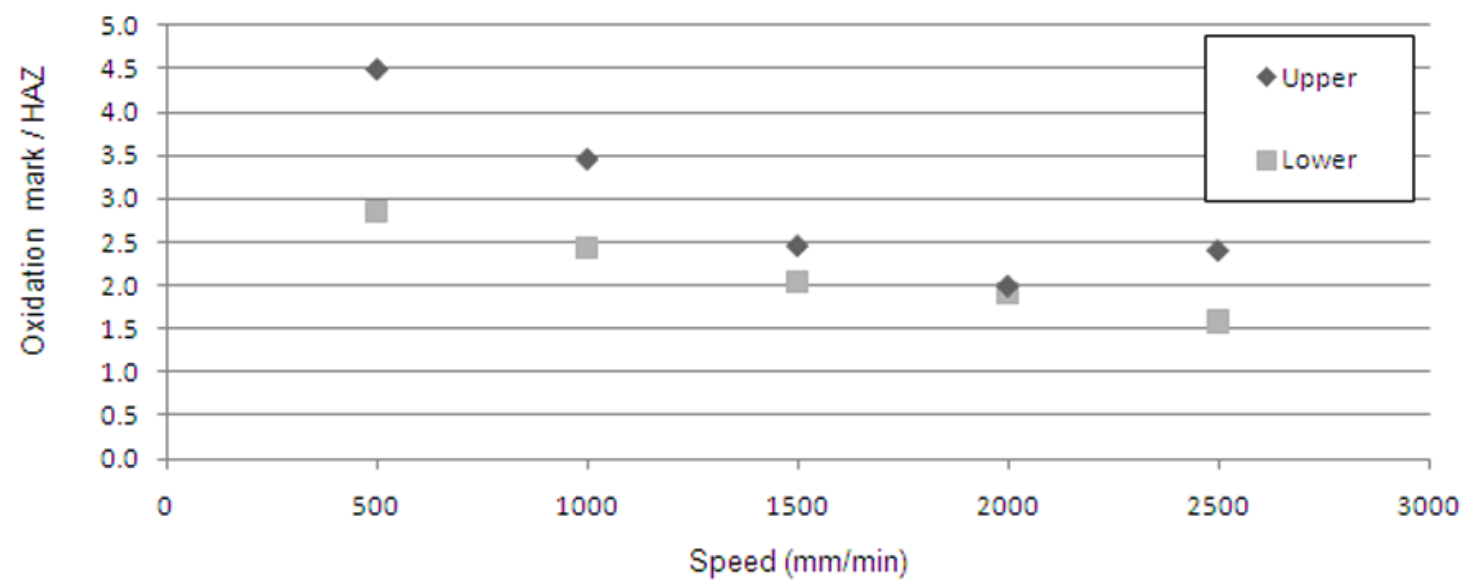

Figure 14 The ratio of the oxidation mark to the $\mathrm{HAZ}$ width for $4.0 \mathrm{~mm}$ mild steel.

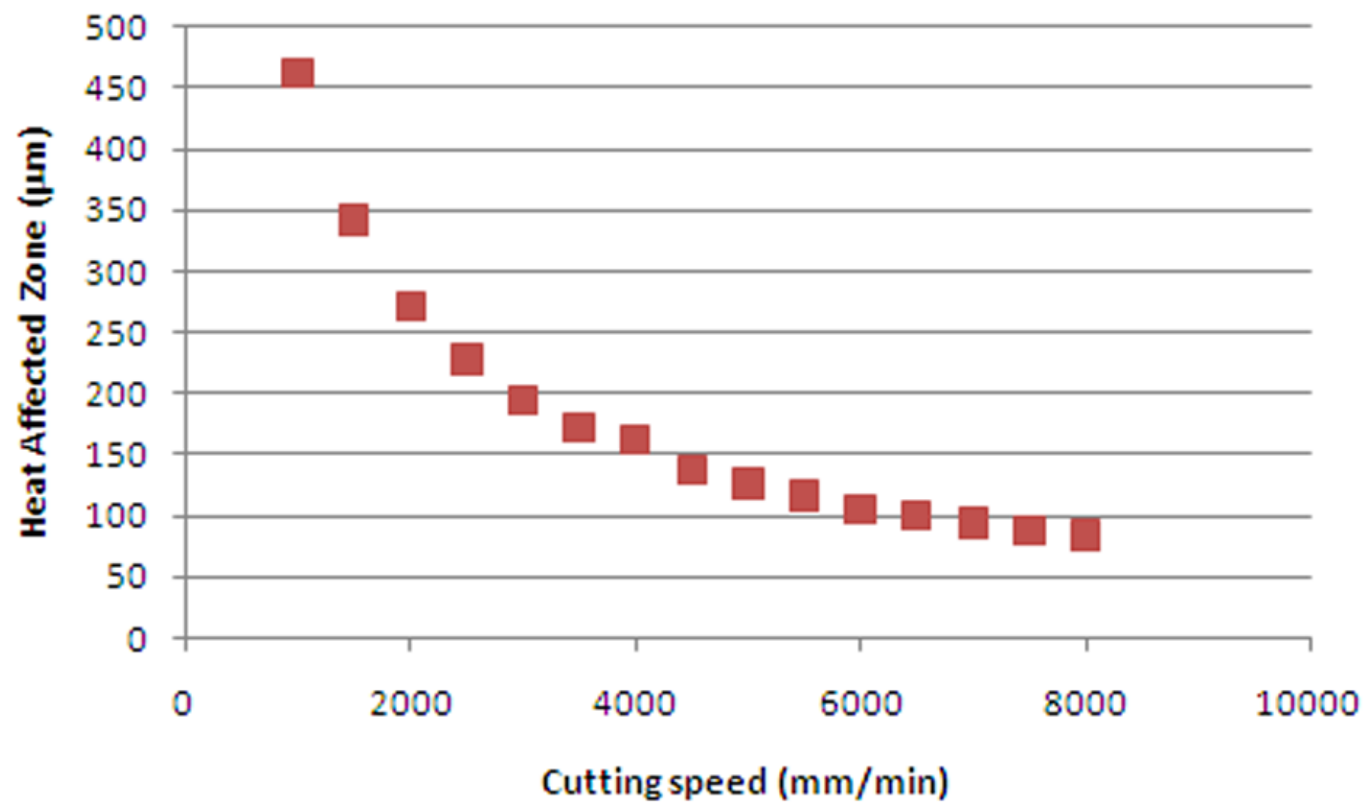

Figure 15 Modelled HAZ width for $\mathbf{2 m m}$ mild steel at $\mathrm{z}=\mathbf{0}$. 

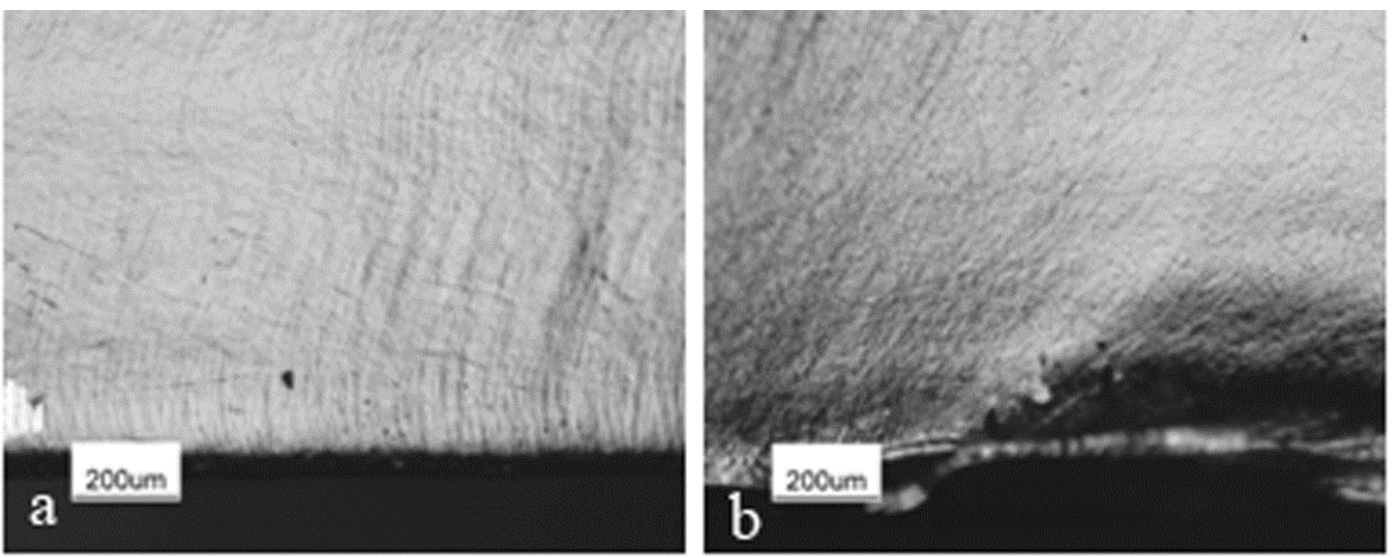

Figure 16 a. A cut at moderate speed $\left(5000 \mathrm{~mm} \mathrm{~min}^{-1}\right)$ with no dross, $b$. A cut edge at higher speed $\left(5500 \mathrm{~mm} \mathrm{~min}^{-1}\right)$ showing dross on the bottom of the cut edge. Laser power $1000 \mathrm{~W}$, material thickness $2 \mathrm{~mm}$. 